\title{
Das Wasser der Spree innerhalb der Stadt Berlin im Jahre 1886 und im Jahre 1896 in bakteriologischer und chemischer Beziehung.
}

Vou

Dr. med. Georg Frank

in Wiesbaden.

Im Jahre 1886/87, in der Zeit vom 4. April 1886 bis zum 2. März 1887, habe ich, damals Assistent am hygienischen Institut der Unirersität Berlin, unter Leitung des Hrn. Geh. Rath Prof. Dr. R. Koch das Wasser der Spree innerhalb und unterbalb Berlin an 22 Tagen chemisch und bakteriologisch untersucht. Ueber die dabei gewonnenen Resultate habe ich im Jahre $1888^{1}$ berichtet. 10 Jahre später haben zwei Assistenten desselben Instituts, jetzt unter Leitung des Hrn. Geh. Rath Prof. Dr. Rubner, die Herren Stabsarzt Dr. Dirksen und Dr. Oscar Spitta diese Untersuchungen wiederholt. ${ }^{2}$

Bei dieser zweiten Untersuchung wurden die Proben an denselben Stellen und auch an denselben Tagen - mit einer Ausnahme 1886, 27. Juli; 1896, 28. Juli - wie bei der ersten entnommen. Ein zweiter Unterschied betrifft die Zahl der gemachten Untersuchungen: bei der ersten wurden 22 - 19 im Jahre 1886, 3 im Jahre 1887 -, bei der zweiten wurden nur halb so viele Untersuchungen, 11 - in der Zeit vom 14. Juli bis zum 11. December $1896-$, gemacht.

In den 10 Jahren von 1886 bis 1896 haben sich die Dinge an der Spree, sowohl oberhalb wie innerhalb der Stadt Berlin, sehr verändert. Die Bevölkerung in den Vororten Berlins, zwischen Köpenick und Oberbaumbricke, ist gewaltig angewachsen. Die Zahl der industriellen An-

1 Diese 2eitschrift. Bd. III. S. 355-404.

${ }^{2}$ Arehiv für Hygiene. Bd. XXXV. S. 85-135 
lagen - und wohl auch der Vergnügungs-Etablissements - hat stark zugenommen. Das Canalisationswerk der Stadt Berlin ist seitdem sehr vorgeschritten, seiner Beendigung beinahe entgegengeführt, soweit bei einer so rasch und so starl sich vermehrenden Stadt, wie Berlin, von der Vollendung einer Anlage, an die täglich neve Ansprüche herantreten, überhaupt geredet werden kann. Das Strombett der Spree ist in dieser Zeit einer gründlichen Reinigung und Correctur unterzogen worden. Der Flussverkehr hat sehr stark zugenommen; neue Bauten und Anlagen, die demselben dienen, sind an den Ofern entstanden.

Dass unter diesen veränderten Verhältnissen das Wasser der Spree im Jahre 1896 eine andere Beschaffenheit zeigen könnte, als im Jahre 1886, mag Nanchem von vornherein als sehr wahrscheinlich erscheinen. Eine Untersuchung des Spreewassers zur Beurtheilung dieser Frage, ob die veränderten Verhältnisse auf die Beschaffenheit des Wassers einen Einfluß ausgeübt hätten, und in welcher Richtung dieser Einfluss sich geltend gemacht habe, war also gewiss eine dankbare und dankenswerthe Aufgabe. Damit haben sich Dirksen und Spitta nicht begnügt, sie sind weiter gegangen und haben die Schlüsse, welche ich aus meinen Untersuchungen des Jahres 1886/87 zog, mit den ihrigen verglichen und geprüft. Sie sind dabei zu Anschauungen und Folgerungen gekommen, welche die meinigen als irrig erweisen sollen.

Dies war für mich die Veranlassung, meine Arbeit an der Hand der von Dirksen und Spitta gemachten Einwände zu prüfen, gleichzeitig aber auch an ibre eigene Arbeit die britische Sonde anzulegen.

Nein Urtheil über den Zustand der Spree im Jahre 1886/87 war folgendes: Die Spree betritt Berlin in schon verunreinigtem Zustande. Die Verunreinigung nimmt innerhalb Berlin sehr stark zu. Hierbei zeigt sich der Unterschied, dass die Verunreinigung im Hauptstrome der Spree, wenngleich an und für sich sehr stark, doch in einem geringeren Massse als in dem Nebenarme, dem sogenannten Landwehrcanale, stattfindet. Den Hauptgrund der Verunreinigung des Stromes erkannte ich in dem Zustande der anlagernden, nicht oder nur theilweise canalisirten Stadttheile, die, wie ich vermuthete, einen Theil ihrer Abwässer in die Spree einleiteten. Des weiteren war ich der Ansicht, dass auch den Nothauslässen, sowohl den selbstthätigen wie auch den regulirbaren, ein gewisser Einfluss zuzusprechen sei. Ein drittes Moment der Verunreinigung sah ich in dem Schiffsverkehr auf der Spree. Hierbei dachte ich nicht allein an den Lösch- und Ladeverkehr, an das auf der Spree, besonders innerhalb Berlin, übliche Vorwärtstreiben der Schiffe mit Stangen, das den auf dem Boden abgesetzten Schlamm aufrührt und wieder in die Höhe bringt, sondern auch an das Leben und Treiben der 
Schiffsbevölkerung, die allen Unrath, alle Schmutzwässer direct in den Fluss abstösst.

Dirksen und Spitta ziehen ausihren Untersuchungen folgendeSchlüsse: Eine Verunreinigung des Spreewassers innerhalb Berlin findet auch beute noch, und zwar in nicht geringerem Grade wie 1886 statt. Die Ursache dieser Verunreinigung war im Jahre 1886 ebenso wie im Jahre 1896 ausschliesslich der Lösch- und Ladeverkehr der Schiffe. All' die Abwässer, welche aus der Stadt (Nothauslässen) oder von den Schiffen in die Spree hineingelangen Łönnen, sind ganz harmlose Dinge, die träben kein Wässerchen.

Prüfen wir nun die Beweise, welche Dirksen und Spitta für ihre Anschauungen beibringen. Dies allein genügt aber nicht, um die Kritik, welche beide Autoren an meinen und ihren Untersuchungen üben, klarzulegen, sondern wir müssen auch die Weise beleuchten, in welcher sie eigene und andere Untersuchungsresultate zusammenfassen.

In einer Tabelle ${ }^{1}$ stellen Dirksen und Spitta die Resultate ihrer bakteriologischen Untersuchungen aus dem Jahre 1896 und der meinigen aus dem Jahre 1886 zusammen. In ihrer Aufstellung haben sie die in den beiden Jahren an den entsprechenden Entnahmestellen bestimmten Keimmengen untereinandergeschrieben; ich' werde die Zahlen getrennt aus dem Jahre 1886 und 1896 in zwei Tabellen (Seite 190) neben einander stellen.

Betrachtet man zuerst jede der beiden Tabellen für sich, so erkennt man auf den ersten Blick, dass im Jahre 1886 die Menge der Bakterien des Spreewassers von der Oberbaumbrücke an von Entnahmestelle zu Entnahmestelle ${ }^{2}$ sehr stark zunimmt. Die Untersuchungen rom 17. November 1886 und 1. December 1886 machen hiervon eine Ausnahme, insofern als an diesen beiden Tagen das Spreewasser innerhalb Berlin an mehreren Stellen geringere Keimmengen mit sich führte, als an der Oberbaumbrücke. Diese Abnahme ist an und für sich nur unbedeutend und durchaus unregelmässig (vgl. auch Tabelle II, S. 194). Die in jenen 11 Untersuchungen festgestellte Vermehrung der Keime innerhalb Berlin erfolgt jedoch nicht regelmässig; dieselbe nimmt nicht ron Entnahmestelle zu Entnahmestelle in gleichmässigem Schritte zu, sondern sie geht sprunghaft ror; auch kommt es gar nicht selten vor, dass an unterhalb gelegenen Orten die Keimmengen geringer waren, als an höher aufwärts gelegenen. Die Verunreinigungen erfolgten also an den verschiedenen Orten und an den verschiedenen Tagen in durchaus ungleicher Stärke. Trotz dieser Tnregelmässigkeiten ist aber die ansteigende Tendenz nicht zu verkennen. Ein anderes Bild geben die Zahlen aus dem Jahre 1896. Zuerst nimmt die Menge der Keime durchaus nicht mehr in so starkem

\footnotetext{
${ }^{1}$ Archix für Hygiene. Bd. XXV. S. 105.

${ }^{2}$ Vgl. Diese Zeitschrift. Bd. III. Tafel IX.
} 

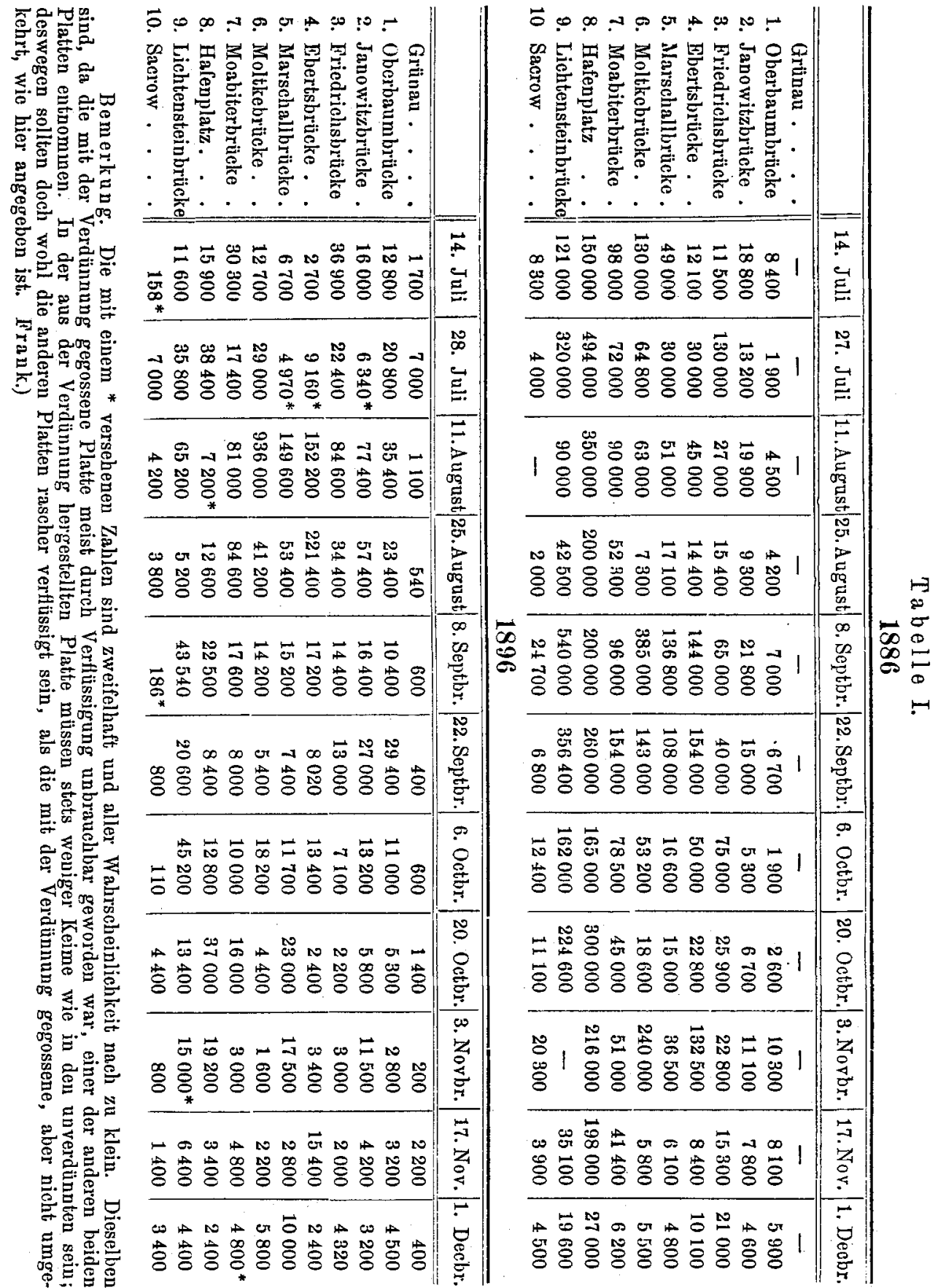
Maasse von Entnahmestelle zu Entnahmestelle zu; auch constatiren wir sehr viel häufiger an unterhalb gelegenen Entnahmestellen geringere Keimmengen als an der Oberbaumbrücke. An einem Tage, am 22. September, nimmt sogar die Keimmenge von der Oberbaumbrücke an bis zur Moltkebrücke regelmässig $a b$ und zeigt nur an der Moabiterbrücke eine geringe Steigerung, die aber auch noch unter der Höhe der Bakterienmenge an der Oberbaumbrücke zurückbleibt. Von den übrigen Untersuchungen weicht auch das Resultat der Untersuchung vom 11. August sehr stark ab. Schon an der Oberbaumbrücke enthält das Wasser an diesem Tage sehr viele Keime. Bei keiner der anderen 10 Untersuchungen des Jahres 1896 wurde nochmals eine gleich grosse Keimmenge bestimmt. Trotzdem nimmt dieselbe stetig von Entnahmestelle zu Entnahmestelle zu und erreicht an der Moltkebrücke die ganz aussergewöhnliche Höhe von 936000 Keimen in $1{ }^{\mathrm{cm}}$ Wasser. Vergleichen wir mit dieser einen Zahl die Bakterienmenge der 10 anderen an der gleichen Entnahmestelle geschöpften Wasserproben, so finden wir die nächsthöchste am 25. August. Aber auch diese ist nur ${ }^{1 / 22}$ der vom 11. August (41 200:936 000); bei den 9 übrigen Untersuchungen schwankt die Keimmenge zwischen 1600 bis 29000 Keime in 1 com Wasser. Zählt man die Werthe der 10 Untersuchungen, mit Ausnahme der vom 11. August, zusammen, so erbält man die Zahl 134700. 134700 ist beinahe der 7. Theil von 936000 . Diese eine Untersuchung rom 11. August zählt also etwa ebenso viel wie 70 sonstige zählen würden. Diese gunz abnorm hohe Zahl verdient unsere ganz besondere Beachtung. Dirksen und Spitta haben das nicht erkannt. Dies erklärt einen Theil ihrer irrigen Vorstellungen und falschen Urtheile. Auch die Untersuchung vom 25. August zeigt zum Theil ähnliche, wenn auch nicht so auffallende Abnormitäten.

Gehen wir nun dazu über, das Verhalten des Spreewassers an den verschiedenen Entnahmestellen an denselben Tagen im Jahre 1886 und 1896 mit einander zu vergleichen.

Das Wasser der Spree enthielt an der Oberbaumbrücke im Jahre 1896 an 8 Untersuchungstagen mehr Keime als im Jahre 1886. Die drei Tage, in denen im Jahre $1896^{\circ}$ die Keimmenge geringer war als im Jahre 1886, fallen auf die Zeit rom 3. November bis zum 1. December 1896. An diesen Tagen war das Spreewasser überhaupt, im Vergleich zu den vorhergegangenen Wochen des Jahres 1896, relativ arm an Bakterien. An der nächstfolgenden Stelle der Janowitzbrücke hat sich das Verhältniss schon geändert. Nur noch an 5 Untersuchungstagen des Jahres 1896 ist der Keimgehalt höher als an den entsprechenden Tagen des Jahres 1886, an 6 dagegen geringer. An den beiden folgenden Entnahmestellen, der Friedrichsbrücke und der Ebertsbrücke, war der Keimgehalt nur noch 
an 3 Untersuchungstagen des Jahres 1896 höher, an 8 aber niedriger als an den entsprechenden des Jahres 1886. An der Marschallbrücke finden wir dagegen eine kleine Steigerung: 4 Mal ist an den entsprechenden Tagen des Jahres 1896 die Keimzahl höher als im Jahre 1886. Bei der nächsten Stelle sinkt das Verbältniss wieder, nur an 3 Untersuchungstagen des Jahres 1896 wurde eine höhere Keimmenge als an den entsprechenden des Jahres 1886 gezählt. An der letzten Entnahmestelle im Hauptstrome, an der Moäbiterbrücke, ist die Keimmenge nur noch an einem einzigen Untersuchungstage höher als an dem entsprechenden des Jahres 1886. Im Landwehrkanal aber an beiden Entnahmestellen, dem Hafenplatz und der Lichtensteinbrücke, bleibt bei allen 11 Untersuchungen im Jahre 1896 der Keimgehalt hinter dem des Jahres 1886 zurück.

Aus dem Vergleiche der bakteriologischen Untersuchungen der Jahre 1886 und 1896 ziehen Dirksen und Spitta folgende Schlüsse. Auf S. 107 sagen sie: „So zeigt sich, wenn die Stationen nach der Reihenfolge, wie sie flussabwärts folgen, betrachtet werden, dass an der 0 berbaumbrücke während unserer Beobachtungszeit ein nicht unerheblich grösserer ${ }^{1}$ Keimgehalt bestand, als im Jahre 1886, während an der Janowitzbrücke der Unterschied sich schon verwischt." Den ersten Theil dieses Satzes kann auch ich anerkennen; der zweite Theil desselben aber giebt die thatsächlichen Befunde nicht scharf genug wieder. Es kommt darin nicht zum Ausdruck, dass eine wirkliche Aenderung im Jalıre 1896 gegenüber dem Zustande im Jahre 1886 - unter Berücksichtigung der Bakterienmenge - stattgefunden hat.

Und weiter unten, auf derselben Seite, sagen Dirksen und Spitta: "An den anderen Entnahmestellen im Hauptlauf der Spree ist der Keimgehalt so wechselnd, dass von einer wesentlichen Aenderung, d. h. Besserung gegen früher wohl nicht die Rede sein kann, während eine durchgängige, ganz auffallende Herabsetzung der Keimzahlen an den beiden Entnahmestellen im Landwehreanal zu constatiren ist." Von diesem Satze kann ich dies Mal bloss den zweiten Theil unterschreiben, während ich die Richtigkeit des ersten bestreiten muss. Denn wie ich oben (S. 191 u. 192) gezeigt habe, treten an sämmtlichen Entnahmestellen unterhalb der Janowitzbrücke sehr wesentliche Aenderungen auf, und zwar solche der Verminderung der Keimmenge, die von Dirksen und Spitta auch als eine Besserung angesehen werden.

Im Gegensatze zu Dirksen und Spitta gelange ich also auf Grund desselben Zahlenmateriales $z u$ folgenden Folgerungen:

1 Gesperrt gedruckt ist bloss das, was auch Dirksen und Spitta auf diese Weise haben hervorheben wollen. 
I. Die Spree tritt im Jahre 1896 mit Bakterien reicher beladen in die Stadt Berlin ein, als dies im Jahre 1886 der Fall war. Als die Ursachen dieses stärkeren Bakteriengehaltes im Jahre 1896 erachte ich die Zunahme der Bevölkerung in den Ortschaften am Oberlaufe der Spree zwischen Cöpenick und Oberbaumbrücke, die Vermehrung der Fabriksanlagen - wohl auch der Vergnügungs-Etablissements, welche Abwässer in die Spree einleiten können, die Steigerung des Schiffsverkehrs und wohl auch den Zulauf von Drainwässern aus den nördlichen Rieselfeldern. ${ }^{1}$

II. Im Jahre 1886 nimmt die Mienge der Bakterien im Spreewasser innerhalb Berlin sehr stark zu, und zwar im Landwebrcanal noch vielmehr als im Hauptstrome. Im Jahre 1896 zeigt sich eine deutliche, unverkennbare Besserung des Spreewassers innerhalb Berlin in bakteriologischer Beziehung. Wenngleich auch in diesem Jahre noch die Keimmenge innerhalb der Stadt im Allgemeinen zunimmt, so ist diese Vermehrung nicht mehr so gross und so regelmässig wie im Jahre 1886.

Merkwürdiger Weise ist an einem Untersuchungstage - dem 22. September 1896 - der Keimgehalt des Spreewassers an allen anderen Entnahmestellen innerhalb Berlin sogar geringer als an der Oberbaumbrücke. An diesem Tage findet also in der Stadt Berlin selbst eine Reinigung der oberhalb verunreinigten Spree statt.

III. Im Jahre 1896 wird die Spree oberhalb Berlin mehr, innerhalb Berlin aber weniger stark verunreinigt, als dies im Jahre 1886 der Fall war. Es ist also eine thatsächliche Besserung dieser Verhältnisse eingetreten.

IV. Das Wasser der Spree bei Sacrow, nach dem Durchgange durch die Havelseeen, enthielt im Jahre 1886 in fast allen Untersuchungen weniger Keime als an den weiter aufwärts gelegenen Entnahmestellen; jedoch erreichte dieser Rückgang nicht immer die Keimmenge an der Oberbaumbrücke. Im Jahre 1896 dagegen enthielt das Wasser bei Sacrow in allen Untersuchungen weniger Keime als an der Oberbaumbrücke.

Es hängt dies damit zusammen, dass die Spree sowohl innerhalb Berlin als auch unterhalb (wofür zwei im Jahre 1897 gemachte bakteriologische Untersuchungen sprechen) im Jahre 1896 weniger stark verunreinigt wurde, als im Jahre 1886.

V. Es wurde also die Spree im Jahre 1896 oberhalb Berlin mehr, innerhalb und unterhalb Berlin aber weniger stark verunreinigt als im Jahre 1886.

${ }^{1}$ Seit meinem Weggange von Berlin in März 1888 sind mir die dortigen Verhältnisse ziemlich fremd geworden. Um ein begründetes Urtheil darüber abgeben zu können, in welchem Umfange jede dieser Möglichkeiten in Wirklichkeit an dieser Keimvermehrung hetheiligt ist, gehört eine genaue Kenntniss aller einschlägigen Verbältnisse, die nur am Orte selber gewonnen werden kann, über die jch also nicht verfüge. Die Reihenfolge, in der diese Möglichkeiten genannt sind, ist also rein willkürlich and soll durchaus nichts der wirklichen Reihenfolge der Dinge präjudiciren. 
Die in obigen Tabellen angeführten Zahlen sind sicherlich etwas kraus und mag die Handhabung derselben Manchem unbequem sein. Ich habe dieselben deswegen in der Weise umgerechnet, dass ich die an jedem einzelnen Untersuchungstage bei der Oberbaumbrücke gefundene Keimmenge als Einheit angenommen, zum Ausdrucke derselben die Zahl 100 gewählt und die übrigen Zahlen auf diese Einheit, die Zahl 100, umgerechnet habe.

Tabelle II.

1886

\begin{tabular}{|c|c|c|c|c|c|c|c|c|c|c|c|c|c|}
\hline & & & $\begin{array}{l}\vec{\Xi} \\
\Xi \\
\end{array}$ & 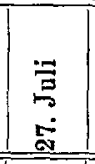 & 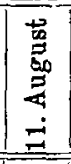 & 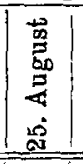 & 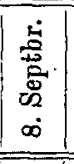 & 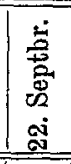 & 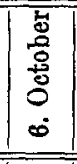 & $\begin{array}{l}\overline{8} \\
\stackrel{0}{0} \\
\stackrel{8}{0} \\
0 \\
0 \\
0\end{array}$ & 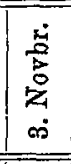 & 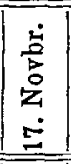 & $\begin{array}{l}\dot{\vec{s}} \\
\stackrel{8}{0} \\
\dot{0} \\
\dot{0}\end{array}$ \\
\hline Oberb & 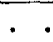 & & 100 & 100 & 100 & 100 & 100 & 100 & 100 & 100 & 100 & 100 & 100 \\
\hline Jan & . & & 224 & 695 & 422 & 225 & 311 & 194 & 279 & 258 & 111 & 96 & 81 \\
\hline cke & . & & 138 & 6947 & 600 & 366 & 928 & 597 & 3945 & 996 & 221 & 189 & 356 \\
\hline & & & 144 & 1579 & 1000 & 3502 & 2057 & 2149 & 2684 & 877 & 1283 & 103 & 171 \\
\hline & & & 595 & 1579 & 1133 & 4071 & 1954 & 1612 & 874 & 577 & $\mathbf{3 5 4}$ & 77 & 81 \\
\hline & & & 1547 & 3410 & 1400 & 1735 & 5500 & 2134 & 2800 & 715 & 2330 & 72 & 93 \\
\hline & & . & 1166 & 3789 & 2000 & 1245 & 1371 & 2149 & 4135 & 1738 & 495 & 511 & 105 \\
\hline & & & 1786 & 26000 & 7777 & $\left.4761\right|_{2}$ & 2857 & 3880 & 8684 & 11537 & 2096 & 2444 & 457 \\
\hline Lichtensteinbrücke & & & 1440 & 17210 & 2000 & $1011 ?$ & 7714 & 5319 & 8526 & 8638 & - & .433 & 332 \\
\hline Sacrow . : . & & & 99 & $|210|$ & 93 & 47 & 353 & 101 & 653 & $\mid 169$ & 197 & 48 & 76 \\
\hline
\end{tabular}

1896

\begin{tabular}{|c|c|c|c|c|c|c|c|c|c|c|c|c|c|}
\hline & & & 访 & $\begin{array}{l}: \bar{\Xi} \\
\stackrel{0}{0} \\
\stackrel{0}{0}\end{array}$ & 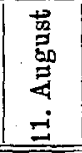 & 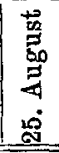 & $\begin{array}{l}\text { 离 } \\
\text { 莒 } \\
\dot{\infty} \\
\infty \\
\infty\end{array}$ & 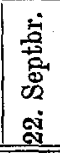 & $\begin{array}{l}\dot{8} \\
\text { 营 } \\
0 \\
0 \\
0\end{array}$ & $\begin{array}{l}\dot{0} \\
\text { 苛 } \\
0 \\
\dot{0}\end{array}$ & 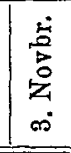 & $\begin{array}{l}\frac{\dot{a}}{2} \\
\frac{2}{2} \\
\stackrel{5}{ \pm}\end{array}$ & 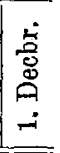 \\
\hline Oberl & & & 100 & 100 & 100 & 100 & 100 & 100 & 100 & 100 & 100 & 100 & 100 \\
\hline & & & 123 & $30^{*}$ & 219 & 145 & 155 & 92 & 120 & 109 & 401 & 131 & 7 \\
\hline & . & . & 288 & 107 & 239 & 142 & 138 & 44 & 64 & 41 & 107 & 63 & 96 \\
\hline & - & . & 21 & $44^{*}$ & 429 & 945 & 165 & 27 & 131 & 45 & 121 & 481 & 53 \\
\hline icke & . & . & 52 & $24^{*}$ & 423 & 228 & 146 & 25 & 106 & 453 & 625 & 87 & 222 \\
\hline & - & . & 99 & 139 & 2813 & 176 & 186 & 15 & 165 & 83 & 56 & 68 & 129 \\
\hline terbrücke & . & 0 & 236 & 83 & 228 & 361 & 165 & 27 & 91 & 302 & 106 & 150 & 107 \\
\hline Hafenplatz . & $\theta^{\circ}$ & . & $124^{*}$ & 184 & $23^{*}$ & 54 & 216 & 28 & 116 & 698 & 686 & 106 & 53 \\
\hline Lichtensteinbrücke & & & 99 & 172 & 184 & 22 & 419 & 72 & 411 & 253 & $536 *$ & 200 & 98 \\
\hline Sacrow . . . & & & 12 & 34 & 11 & 16 & $1 \cdot 8^{*}$ & $2 \cdot 7$ & $1 *$ & 83 & 28 & 47 & 78 \\
\hline
\end{tabular}

Auf S. 112 sagen Dirksen und Spitta folgendes: „Wenn wir nun also als ausgemacht ansehen, dass nur in seltenen Fällen die Canalisation durch ihre Nothauslässe eine Verschmutzung der öffentlichen Wasserläufe verursacht, so drängt sich uns die Frage auf, welche anderen $\mathrm{Um}$ stände wohl die Hauptschuld an der Verunreinigung der 
Wasserläufe tragen und besonders, wodurch gerade an bestimmten Stellen, z. B. an der Moltkebrücke, eine so starke Steigerung des Bakteriengehaltes regelmässig verursacht wird. Wie oben schon erwähnt, führt G. Frank letzteren Umstand darauf zurück, dass damals der Stadttheil Moabit noch nicht canalisirt war, und dass gerade bei jener Brūcke ein grosser alter Canal mündet, eine Ansicht, die uns jetzt als unhaltbar erscheint, nachdem dort die Canalisation durchgeführt ist. Vergleicht man die graphische Darstellung unserer Keimzählungen in Anlage 2 mit den gleichfalls dort eingetragenen Ergebnissen der Frank'sehen Untersuchung, so kommt man zu dem interessantenErgebniss, dass der Charakter beider Curven genau der gleiche ist, indem dieselben je einen kleineren Gipfel bei der Ebertsbrücke und einen erheblich grösseren bei der Moltkebrücke aufweisen."

Dirksen und Spitta haben also aus den bei ibren Untersuchungen gewonnenen Zahlen sogenannte Mittelwerthe berechnet. Dies zu thun, tabe ich bei meiner Arbeit unterlassen, und zwar mit vollster Absicht, weil ich nämlich der Ansicht bin, dass Mittelwerthe nur dann richtig sind, wenn sie aus einer wirklich grossen Reihe von möglichst gleichmässigen Einzelzahlen gewonnen werden. Denn nur eine grosse Zahlenreihe vermag die Fehler der einzelnen Zahlen auszumerzen. Unter einer grossen Zahlenreihe, aus der annähernd richtige Mittelwerthe herausgerechnet werden können, verstehe ich aber nur eine solche von mindestens mehreren Hunderten oder besser noch mehreren Tausenden von Einzelzahlen. Die Statistik hat nur dann Werth, wenn sie mit Kritik und auf Grund grosser Zahlenreihen geübt wird. Eine Statistik mit kleinen Zahlen ist eine müssige Spielerei und häufig noch etwas Schlimmeres, sie ist eine Tăuschung.

Aus meiner Arbeit standen mir für jede Entnahmestelle 22 einzelne Zahlen für jede Bestimmung zur Verfügung. Dirksen und Spitta haben nur 11 Untersuchungen gemacht. 11 und 22 aber sind nach meiner Ansicht bescheidene Zahlen. Dirksen und Spitta sind entgegengesetzter Ansicht. Auf S. 117 sagen sie nämlich:

„Eine Betrachtung der in der Anlage 1 zusammengestellten Ergebnisse der chemischen Untersuchung zeigt, dass sich - was die erste Frage anlangt - irgend eine Regelmässigkeit nicht erkennen lässt. Die Differenzen der Werthe sind relativ so geringe, dass uncontrolirbare accidentelle Umstände bei der Wasserprobeentnahme u. A. m. den wahren Sachrerhalt leicht verdecken können. Unter solchen Umständen empfiehlt es sich, die Durchschnittszahlen zu berechnen, da man hoffen kann, dass sich bei ihnen die kleinen Zufälligkeiten compensiren und ausgleichen werden." 
Und weiter S. 119:

„Die Berechnung der Durchschnittswerthe und deren Zusammenstellung liess aber schon eine gewisse Regelmässigkeit erkennen. Frank hat es nun unterlassen, solche Durchschnittswerthe $z u$ berechnen und wir haben uns deswegen nachträglich dieser Aufgabe unterzogen. Einmal wollten wir sehen, ob sich aus dem Gewirr seiner Zahlen etwas Greifbares und Gesetzmässiges extrahiren liesse und dann wünschten wir seine Resultate mit den unseren besser vergleichen zu können."

Nachdem wir also diese Ansichten kennen gelernt haben, wollen wir dazu übergehen, nachzusehen, wie Dirksen und Spitta rechnen und was. bei dieser Art von Rechnerei herauskommt.

Dirksen und Spitta haben ganz mechanisch die an jeder Entnahmestelle bei den Untersuchungen bestimmten Zahlen unter einander gestellt, addirt und diese Summe durch die Zahl 11 dividirt; den so gefundenen Werth nennen sie dann eine Mittelzahl. Ein Zweifel, ob die entsprechenden 11 Zahlen auch unter einander gleichwerthig sind und deswegen ohne Weiteres mit einander verglichen werden können, kommt ihnen nicht auf. Auf S. 191 'u. 192 habe ich schon darauf aufmerksam gemacht, dass die einfache Vergleichung der an den verschiedenen Untersuchungstagen des Jahres 1896 bestimmten Keimmengen sehr grosse Differenzen ergiebt. und also darauf hinweist, dass auch sehr verschiedene Umstānde mitgewirkt haben müssen. Bei 9 von den 11 Untersuchungen konnten wir eine Vermehrung der Bakterienmenge des Spreewassers innerhalb Berlin, die häufige Schwankungen zeigte und sich im Allgemeinen innerhalb mässiger Grenzen hielt, erkennen. Bei einer Untersuchung, der vom 22. September, sahen wir das Gegentheil, nämlich, dass das Wasser der Spree innerhalb Berlins allmählich sich reinigt, indem die Menge der Keime von Entnahmestelle zu Entnahmestelle geringer wird. Auch die Untersuchung vom 11. August macht eine Ausnahme von dem gewöhnlichen Verhalten. Ich habe schon auf S. 191 ausführlich darauf hingewiesen, dass an diesem Tage die Menge der Bakterien an den Entnahmestellen Oberbaum-, Janowitz-, Friedrichs-, Eberts-, Marschall-, Moabiter- und ganz besonders Moltkebrüeke höher gewesen ist als an den übrigen Untersuchungstagen. Einen Versuch, diese Verschiedenheiten zu erklären, machen Dirksen und Spitta nicht. Sie begnügen sich damit, ihr an und für sich unbedeutendes und dazu noch ungleichmässiges Zahlenmaterial als vollständig gleichwerthig zu behandeln.

Folgt man also Dirksen und Spitta, addirt man die gefundenen Zahlen bei den bakteriologischen Untersuchungen des Jahres 1896 und dividirt diese Summe durch die Zahl 11, so erhält man folgende Zahlen : 
1. Oberbaumbrücke . . . . 14454

2. Janowitzbrücke . . . . 21643

3. Friedrichsbrücke . . . . 20392

4. Ebertsbrücke . . . . . 40698

5. Marschallbrücke . . . . 27388

6. Moltkebrücke . . . . . 97366

7. Moabiterbrücke . . . . 25172

Rundet man diese Zahlen ab, so ergiebt sich folgende Reihe:

1. Oberbaumbrücke . . 14000

2. Janowitzbrücke . . 22000

3. Friedrichsbrücke . . 20000

4. Ebertsbrücke . . . 41000
5. Marschallbrücke . . 27000

6. Moltkebrücke . . 97000

7. Moabiterbrücke . . 25000

Trägt man diese Zahlen graphisch auf, so erhält man folgende Curve:

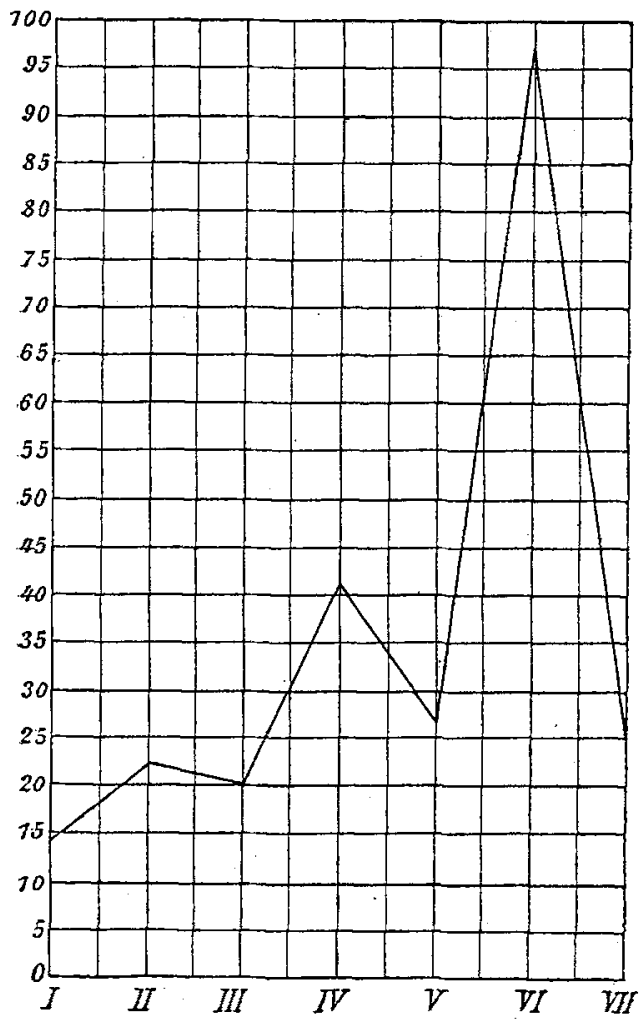

1 Anf dieser und den nächsten Tabellen bedeutet I Oberbanmbrücke, II Janowitzbrücke, III Friedrichsbrücke, IV Ebertsbrücke, V Marschallbrücke. VI Moltkebrücke, VII Moabiterbrücke. 
Lässt man aber die beiden Untersuchungen vom 11. August und 22. September aus der Berechnung weg, weil beide, wie nachgewiesen, ganz aussergewöhnliche, von Dirksen und Spitta nicht gewürdigte Verhältnisse zeigen, so erhält man folgende Zahlenreihe:

1. Oberbauınbrücke . . . . 12680

2. Janowitzbrücke . . . . 14893

3. Friedrichsbrücke . . . . 11864

4. Ebertsbrücke . . . . . 31940

5. Marschallbrücke. . . . . 16189

6. Moltkebrücke . . . . . 14333

7. Moabiterbrücke . . . . . 20944

Rundet man diese Zahlen ab, so ergiebt sich folgende Reihe:

1. Oberbaumbrücke . . . . 13000

2. Janowitzbrücke . . . . 15000

3. Friedrichsbrücke . . . . 12000

4. Ebertsbrücke . . . . . 32000

5. Marschallbrücke . . . . 16000

6. Moltkebrücke . . . . . 14000

7. Moabiterbrücke. . . . . 21000

Trägt man diese Zahlen graphisch auf, so erhält man nachstehende Curve.

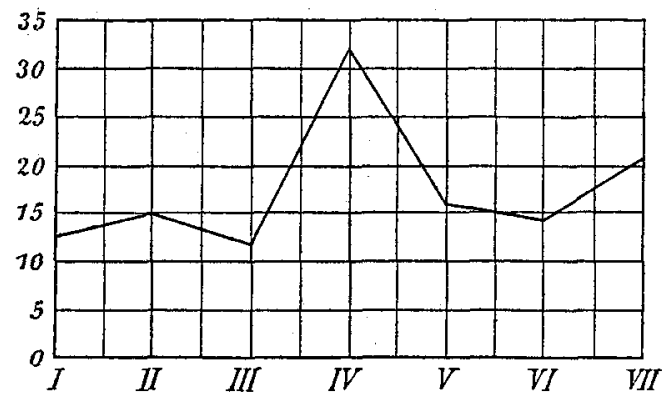

Diese zweite Curve unterscheidet sich von der ersten, welche der von Dirksen and Spitta gegebenen vollständig entspricht, in allen Punkten. Die grössten Unterschiede fallen aber auf die Moltkebrücke und die Moabiterbrücke. In der zweiten Curve ist die Erhöhung an der Moltkebrücke ausgefallen und an deren Stelle eine Senkung getreten, an der Moabiterbrücke dagegen steht eine Spitze an der Stelle einer Senkung.

Ich habe diese beiden Curven nur aus dem Grunde gezeichnet, um zu zeigen, was man aus einem so unbedeutenden Materiale alles heraus- 
rechnen kann und weiche Unterschiede sich ergeben, je nach der Art, wie man dasselbe behandelt. Denn ich halte diese zweite Curve für mindestens ebenso falsch, wie es die erste sicher ist. Denn auch die Ergebnisse der Untersuchung vom 25. August 1896 weichen von denen der anderen Untersuchungstage $a b$, wenn auch nicht in dem Maasse, wie die der vorhergegangenen Untersuchung. Berechnet man aus 8 Untersuchungen ich bin der Ansicht, dass man dies aus 8 ebenso gut darf wie aus 11 die Mittelwerthe und zeichnet diese graphisch auf, so erhält man eine dritte, von den beiden anderen unterschiedliche Curve, die aber auch um nichts richtiger ist.

An der Moltkebrücke hat also im Jahre 1896 durchaus nicht regelmässig eine Vermehrung des Bakteriengehaltes stattgefunden, wie Dirksen und Spitta behaupten und durch ihre Curve beweisen wollen, sondern es war dies ein ganz ausnahmsweises Ereigniss. Es wäre die Aufgabe von Dirksen und Spitta gewesen, nachzuweisen, durch welche Umstände diese ausnahmsweise Verunreinigung des Spreewassers an der Moltkebrücke am 11. August des Jahres 1896 bewirkt wurde. Ich möchte vermuthen, dass wieder einmal Schmotzwässer aus dem alten Canal ausgeflossen sind.

Ich bin also nicht in der Lage, den Schlüssen, welche Dirksen und Spitta aus diesen bakteriologischen Zahlen gezogen haben, beizutreten; wohl aber glaube ich, dass man auf Grund derselben gewisse Vermuthungen doch aussprechen darf. Ich halte also drei Möglichkeiten der Verunreinigung der Spree im Jahre 1896 innerhalb Berlin für wahrscheinlich:

Erster Zustand: Mässiger Grad der Verunreinigung mit unregelmässigen Schwankungen (11. Juli u. a.).

Zweiter Zustand: Hochgradige und fortschreitende Verunreinigung (11. August, vielleicht auch 25. Angust).

Dritter Zustand: Reinigung des Spreewassers innerhalb Berlin (22. September).

Diese Ansicht spreche ich hier mit allem Vorbehalte und nur als Muthmassung aus, für deren mögliche Richtigkeit die Untersuchungen von Dirksen und Spitta gewisse, nicht zu unterschätzende Andeutungen ergeben haben. Ob überhaupt und in welchem Umfange diese Vermuthungen den thatsächlichen Verhältnissen entsprechen, mïsste durch weitere, während längerer Zeit fortgesetzte Untersuchungen geprüft werden.

Den gleichen Vorwurf, keine Mittelzahlen berechnet zu haben, machen mir Dirksen und Spitta ausser an den schon vorher angeführten Stellen auch mit Bezug auf die Beurtheilung der Resultate der chemischen Untersuchungen. So sagen sie auf S. 120: „Frank, der diese Durchschnittsberechnungen nicht ausgeführt, wird nach unserer Meinung dadurch zu 
falschen Schlussfolgerungen geführt. So behauptet er: ,Der Chlorgehalt der Spree nahm, wie wir oben gesehen haben, im Verlaufe durch Berlin in unregelmässiger, aber deutlich zu erkennender Weise zu." Diese Angabe lässt sich auf Grund des von ihm vorgelegten Zahlenmateriales nicht rechtfertigen."

Gewiss, Dirksen und Spitta haben mit ihrer Behauptung Recht, aber nur, wenn man, wie sie es thun und wie ich es nicht thue, Durchschnittszablen berechnet, welche die Unterschiede der einzelnen Untersuchungen verwischen. Sieht man aber die Originalzahlen selber an, so werden diese kleinen Unterschiede klar und deutlich und bestätigen das, was ich gesagt habe. Ich gebe also im Folgenden eine Tabelle, in welcher ich den Chlorgehalt des Spreewassers an den 22 Untersuchungstagen zusammengestellt habe. Der leichteren Uebersicht wegen sind die steigenden Werthe durch besonderen Druck hervorgehoben.

Es wurden gefunden Milligramm Chlor:

Tabelle III.

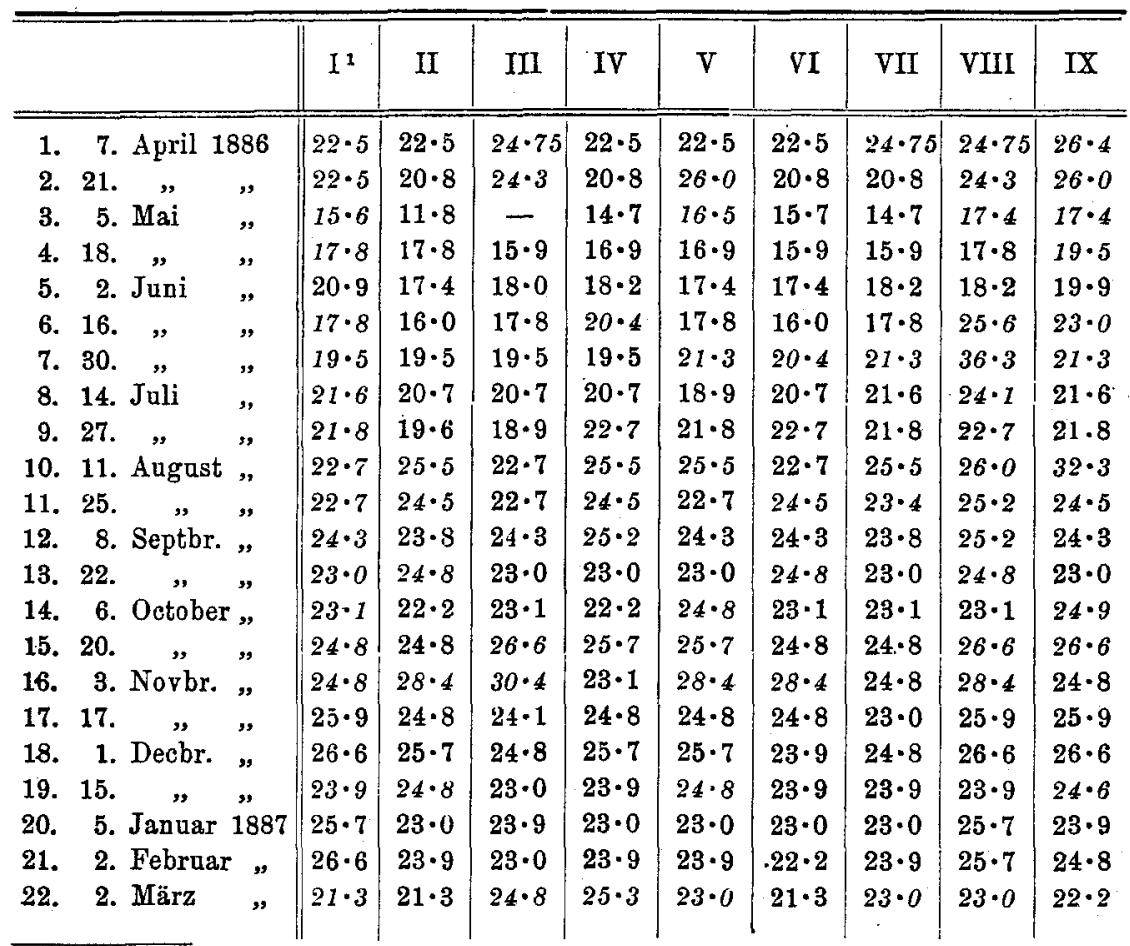

1. Siehe Anmerkung Seite 197. VIII bedentet Hafenplatz, IX Lichtensteinbrücke. 
Diese Tabelle ergiebt deutlichst, dass bei 17 von den an 22 Tagen gemachten Untersuchungen der Chlorgehalt an den Entnahmestellen unterhalb der Oberbaumbrücke höher war, als an der Oberbaumbrücke selber. An den 5 Tagen, an welchen der Chlorgehalt an der Oberbaumbrücke aber höher war als an den unteren Entnahmestellen, müssen deswegen aussergewöhnliche ${ }^{1}$ Umstände vorgelegen haben. Am 2. Juni ist der Chlorgehalt im ganzen Verlaufe der Spree innerhalb Berlin abnorm niedrig; an der Oberbaumbrücke selber ist er auch an diesem Tage an und für sich nur gering und im Vergleiche mit demselben an den sonstigen Untersuchungstagen nur mässig hoch. An den 4 anderen Untersuchungstagen (17. November, 1. December, 5. Januar und 2. Februar) war der Chlorgehalt im ganzen Verlauf durch Berlin ungefähr gleich hoch mit nur geringeu Schwankungen. Das Verhalten des Chlorgehaltes des Spreewassers innerhalb Berlin kann ich auch heute noch, trotz des Widerspruches, den Dirksen und Spitta dagegen erheben, nicht kürzer und präciser zusammenfassen, als in dem von ihnen für falsch erklärten Satze:

Der Chlorgehalt der Spree nahm im Verlaufe durch Berlin in unregelmässiger, aber deutlich zu erkennender Weise zu.

Welche Bedeutung dürfen wir dieser Zunahme des Chlorgehaltes beimessen? Da ich in so vielen Punkten anderer Ansicht bin als Dirksen und Spitta, so freue ich mich ganz besonders, auch eine Aeusserung vou ihnen anführen zu können, die mit meinen Ansichten vollständig übereinstimmt. Auf S. 123 sagen sie: „Da das Steigen der Chlormenge in einem Flusse bekanntlich fast ausschliesslich durch Verunreinigung des Wassers mit Harn und Koth verursacht wird, -." Auf Grund der von mir im Jahre 1886/87 ausgeführten Bestimmungen des Chlorgehaltes können wir also sagen, dass im Jahre 1886/87 menschliche Fäkalien bezw. Flüssigkeiten, welche menschliche Fäkalien mit sich führten, in die Spree innerhalb Berlin hineingelangt sind. Trotz des Widerspruches ron Dirkșen und Spitta im speciellen Falle, aber auf Grund ihrer eigenen allgemeinen Anschauungen beharre ich demnach bei dem Ausspruche, welchen ich im Jahre 1888 gethan habe, dass eine Verunreinigung der Spree durch Fäkalien führende Schmutzwässer im Jahre 1886 stattgefunden hat.

Wie aber verhielt sich in dieser Beziehung die Spree im. Jahre 1896? Dirksen und Spitta sagen auf S. 124: „Das Bine ergeben unsere Untersuchungen jedenfalls mit Sicherheit; Trotz des Ausschlusses der Abwässer Berlins von der Spree und trotz der Verbesserung ihrer Zuflüsse hat dieser

1 In Jahre 1886 war der an die Oberbaumbrücke anlagernde Radialbezirk XII noch nicht an die Rieselfelder angeschlossen. 
Fluss eine Verbesserung seiner Beschaffenheit in bakteriologischer wie chemischer Hinsicht nicht aufzuweisen." Dass sich das Spreewasser im Jahre 1896 in bakteriologischer Beziehung entschieden gebessert hat gegenüber dem Jahre 1886, glaube ich durch meine früheren Ausführungen auf S. 191 und 192 bewiesen zu haben. Eine gleiche Besserung in chemischer Beziehung für das Jahr 1896 geht aber aus den Untersuchungen von Dirksen und Spitta nicht hervor. Zwar behaupten sie S.119, dass sich für das Chlor eine Steigerung der Quantitäten nicht nachweisen lässt. Diesen Schluss ziehen sie wiederum aus Mittelzahlen; verfulgen wir aber das Chlorim Jahre 1896 von Entnahmestelle zu Entnahmestelle, so finden wir an allen 9 Untersuchungtagen, dass an einigen unterhalb gelegenen Entnahmestelle der Chlorgehalt höher war als an der Oberbaumbrūcke. ${ }^{1}$

Tabelle IV.

Gramm Chlor im Liter 1896.

\begin{tabular}{l||l|l|l|l|l|l|l|l|l}
\hline \hline & I & II & III & IV & V & VI & VII & VIII & IX \\
\hline 1. 14. Juli 1896 & 30 & 28 & 31 & 30 & 30 & 29 & 30 & 50 & 35 \\
2. 28. ", " & $\mathbf{3 4}$ & 33 & 32 & 34 & 33 & 35 & 35 & 36 & 34 \\
3. 11. August ", & 33 & 33 & 33 & $\mathbf{3 3}$ & 35 & 34 & 34 & 35 & 36 \\
6. 22. Septbr. ", & 27 & 27 & 30 & 30 & 29 & 27 & 29 & 30 & 33 \\
7. 6. October ", & 27 & 27 & 28 & 26 & 27 & 27 & 27 & 27 & 29 \\
8. 20. ", " & 24 & 25 & 26 & 24 & 25 & 24 & 26 & 27 & 25 \\
9. 3. Novbr. ", & 27 & 26 & 27 & 28 & 28 & 24 & 28 & 29 & - \\
10. 17. ", ", & 22 & 22 & 23 & 22 & 23 & 23 & 23 & 24 & 24 \\
11. 1. Decbr. ", & 24 & 23 & 24 & 23 & 23 & 24 & 23 & 25 & 24
\end{tabular}

Chemische Untersuchungen sind am 25. Augast und 8. Sept. nicht gemacht, worden.

1 Der Fehler, welchen Dirksen und Spitta bei ihrer Arbeit in Folge der Berechnung von Mittelzahlen gemacht haben, ist in der medicinischen und besonders der hygienischen Litteratur durchans nicht so selten. Ich will hier ein ähnliches Beispiel anführen, auf welches ich schon an anderer Stelle (Journal für Gasbeleuchtung und Wasserversorgung, 1898, Nr. 39, S. 633) aufmerksam gemacht habe. In Nr. 4 der Hygienischen Rundschau, 1898, S.161, berichten Goldschmidt, Luxemburger, Franz, Hans und Ludwig Neumayer und W. Prausnitz über bakteriologische Untersuchungen des Isarwassers. Als Hanptmittel aller Untersuchungen geben sie für das Wasser oberhalb München einen Bakteriengehalt von 640 Keimen an. Betrachten wir die bei den einzelnen Untersuchungen gefundenen Keimmengen, so finden wir folgende Zahlen: $97,54,160,34,814,601,2680,928,2239,980$, 132, 50, 120, 20. Unter diesen 14 Zahlen sind 8 bedeutend niedriger, 5 bedeutend höher als die Mittelzahl - 640 - und nur eine kommt derselben nahe. Diese Mittelzahl entspricht meiner Ansicht nach nicht den thatsächlichen Ver- 
Während also Dirksen und Spitta behaupten, dass ihre Untersuchungen ergaben, dass eine Aenderung, d. h. Besserung des Spreewassers im Jahre 1896 gegenüber dem Jahre 1886 weder in bakteriologischer noch in chemischer Hinsicht stattgefunden hat, ziehe ich aus ihren Untersuchungen und dem Vergleiche derselben mit meinen aus dem Jahre 1886 folgende Schlüsse:

1. In bakteriologischer Beziehung ist eine Aenderung, d. h. Besserung des Spreewassers innerhalb Berlins im Jahre 1896 durchaus nicht zu verkennen.

2. In chemischer Beziehung dagegen tritt diese Besserung nicht hervor, insofern als auch im Jahre 1896 noch der Chlorgehalt des Spreewassers innerhalb Berlin zunimmt.

Da die Zunahme des Chlorgehaltes in einem Wasserlaufe nach der Ansicht von Dirksen und Spitta und auch der meinigen darauf hinweist, dass der Fluss Ábgänge aus dem menschlichen Haushalte aufnimmt, so müssen wir also aus dieser Zunahme folgern, dass auch im Jahre 1896 noch Fäkalien in das Spreewasser hineingelangt sind. Sicherlich ist also nicht der Lösch- und Ladererkehr, wie Dirksen und Spitta behaupten, die einzige Ursache der Spreeverunreinigung im Jahre 1896.

Im Jahre 1886 war ein Theil der an die Spree anlagernden Radialsysteme noch nicht ausgebaut; mir erschien es deshalb wahrscheinlich, dass aus diesen ein Theil der von mir constatirten Verunreinigungen herstammen könnte. Ausserdem aber nannte ich noch als verunreinigende Momente die zahlreichen Nothauslässe, welche Schmutzwässer in die Spree einleiten können, und den Schiffsverkehr. Im Jahre 1896 war die Canalisation der Stadt Berlin fast vollendet, nur noch 600 Grundstücke mit nicht ganz 50000 Einwohnern waren nicht an die Rieselfelder angeschlossen. Dass von dieser Seite bedeutende Mengen von fäkalienhaltigen Abwässern in die Spree hineingelangt sind, erscheint durchaus unwabrscheinlich. Denn die betreffenden Stadttheile liegen weit ab von der Spree und sind durch zwischenlagernde, an die Rieselfelder angeschlossene Stadttheile vom Stromlaufe getrennt.

hältnissen und giebt eine durchaus falsche Vorstellung von dem Keimgehalt des Isarwassers oberhalb München. Rïchtiger erscheint mir folgender Ausdruck: In 14 Untersuchungen war der Bakteriengehalt des Jsarwassers oberhalb München 8 Mal niedrig (20 bis $160 \mathrm{Keime}$ ), $4 \mathrm{Mal}$ mässig hoch (601 bis $980 \mathrm{Keime}$ ), $2 \mathrm{Mal}$ sehr stark erhöht (2239 und 2680 Keime). Ein solcher Satz ist wohl eine grössere Belastung des Gedächtnisses als eine einzige Mittelzahl. Dafür' giebt er aber auch eine richtigere Vorstellung der wirklichen Verhältnisse als die Mittelzahl. 
204 Grorg Frank: Das Wasser Der Spree im Jatre 1886 und 1896.

Als ursächliche Momente der Verunreinigung der Spree ${ }^{1}$ im Jahre 1896 sind also nur noch die Nothauslässe und der Schiffsverkehr übrig geblieben. Es wäre eine interessante Aufgabe gewesen, nachzuweisen, in welchem Umfange jeder dieser beiden Factoren im Jahre 1896 an der Verunreinigung der Spree betheiligt gewesen ist. Dirksen und Spitta haben diese Aufgabe nicht erkannt.

${ }^{1}$ Ich habe in dieser Arbeit die Spree innerhalb Berlin einen Flusslauf genannt. Ich bezweifele jedoch, dass diese Bezeichnung richtig ist. Der eine Arm, der Landwehrcanal, ist schon seit langer Zeit ein stagnirendes Wasser; der Hauptstrom aber wird heatigen Tages auch zwei Mal gestaut. An den Dammmühlen findet die erste Stauung statt; bei Charlottenburg unterhalb Berlin ist Ende der 80 er Jahre eine grosse Stauanlage geschaffen. Dirksen und Spitta berücksichtigen in ihrer Arbeit diese Veränderung durchaus nicht. Da mir die Dinge in Berlin fremd geworden sind, ich mich also in meinen Ausführungen bloss auf das stützen kann, was Dirksen und Spitta beibringen, so habe ich dieser Aenderung auch nicht Rechnung tragen können. 